\title{
Serial angiographic findings during the development of a saccular aneurysm of the coronary artery in association with unstable angina
}

\author{
JOHN GOUDEVENOS, MARTIN BEEN, DAVID WILLIAMS \\ From the Cardiothoracic Centre, Freeman Road Hospital, Newcastle upon Tyne
}

SUMMARY A 60 year old man presented with unstable angina and electrocardiographic features suggestive of disease of the left anterior descending coronary artery. Angiography showed slight proximal narrowing in this artery with reduced density of contrast that suggested an associated thrombus. Repeat angiography after 24 hours of heparin administration showed the development of a small saccular aneurysm. Three weeks later, after a further episode of unstable angina, angiography showed enlargement of the aneurysm. Although there were no further episodes of angina, repeat angiography three months after the initial event showed further slight enlargement of the aneurysm.

It is suggested that the episodes of angina at rest were secondary to embolisation of thrombotic or atheromatous material or both.

The finding of single or multiple aneurysmal dilatations of coronary arteries is fairly common in patients with ischaemic heart disease ${ }^{1}$ and is usually referred to as coronary ectasia. Isolated saccular aneurysms are much less common, ${ }^{2}$ although the recent report of similar findings in a small number of patients after percutaneous transluminal coronary angioplasty ${ }^{3}$ suggests that in future the incidence may increase.

There has been speculation about the pathophysiological development of the more common coronary aneurysms ${ }^{45}$ but the opportunities for serial angiographic studies have been limited. Despite the increase in early angiographic studies in unstable angina, there are few opportunities for serial angiographic study because mechanical intervention by percutaneous transluminal coronary angioplasty or operation often follows.

The time course, associated symptoms, and serial angiographic findings in a patient with unstable angina in whom a saccular coronary aneurysm developed without any mechanical intervention are therefore of interest.

\section{Case report}

A 60 year old man with a previous inferior myocar-

Requests for reprints to Dr Martin Been, Department of Cardiology, Freeman Road Hospital, Freeman Road, Newcastle upon Tyne NE7 7DN. dial infarction presented with a typical history of recent onset exertional angina culminating in episodic rest pain. He was monitored in the coronary care unit and an oral calcium antagonist, aspirin, and a $\beta$ blocker were added to his nitrate treatment. The admission electrocardiogram showed evidence of his old inferior infarct but there were no acute changes.

Despite bed rest and medical treatment he continued to have episodic pain associated with $T$ wave inversion in leads V2-V6 but with no increase in the activity of cardiac enzymes. Coronary angiography performed by the Judkins technique showed extensive disease of the right coronary artery but the left anterior descending artery, suspected to be the ischaemia related vessel, had a proximal stenosis of $<50 \%$ of the luminal diameter. There was some reduction in the density of contrast in the proximal left anterior descending artery, suggesting the presence of thrombus adjacent to the mild lesion. Heparin was started and the femoral sheath was left in situ. Repeat angiography at 24 hours showed some increase in contrast density in the proximal left anterior descending artery and the development of a small saccular aneurysm.

No further episodes of myocardial ischaemia occurred, the $T$ waves returned towards normal, and before hospital discharge he performed a graded treadmill exercise test. He completed five minutes and 45 seconds of a standard Bruce protocol, and he 


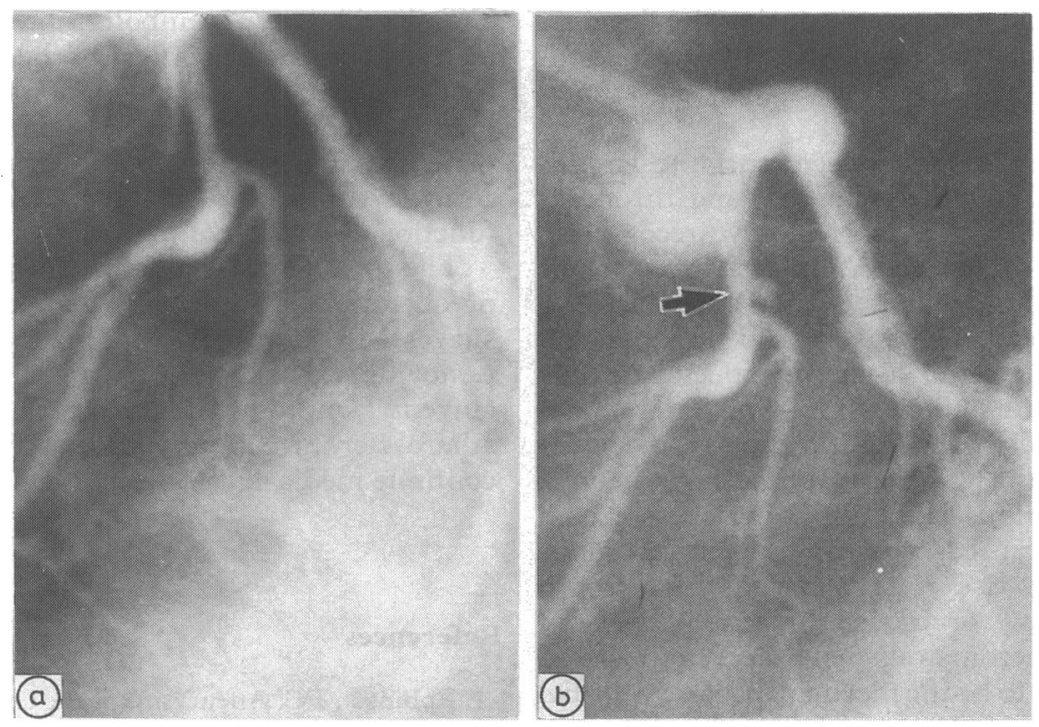

stopped because of dyspnoea. At peak exercise there was $2 \mathrm{~mm}$ ST depression in lead V5. He was discharged on the above medication with the addition of warfarin.

Three weeks later he attended for a radionuclide gated blood pool scan, which showed good left ventricular function with an overall ejection fraction of $41 \%$. He had been free of further angina until the morning of his scan when he was woken by ischaemic pain and developed further rest pain before leaving home. He was readmitted to the coronary care unit and later that day had a further episode of rest pain associated with ST elevation of $1.5 \mathrm{~mm}$ in leads V2 and V3. He responded symptomatically to glyceryl trinitrate but was left with mild residual ST depression and $T$ wave inversion in leads V2-V5. Repeat angiography showed enlargement of the saccular aneurysm with some progression in severity of the proximal coronary stenosis and no convincing evidence of coronary thrombus.

No further clinical events occurred but repeat exercise testing showed evidence of symptom free ischaemia. He completed eight minutes of an identical Bruce protocol and significant ST depression developed in lead V5 by four minutes. Anticoagulants were stopped but other antianginal medication continued.

In view of the exercise test findings and uncertainty as to the development of the aneurysm, a further angiogram was performed three months after the original admission. No further episodes of angina had occurred since his previous hospital admission. Contrast angiography showed that left ventricular function remained good. Coronary angiography showed that there had been some further enlargement of the aneurysm (figure) but there was only slight narrowing of the proximal left anterior descending artery.

\section{Discussion}

Aneurysms are described as fusiform when they are spindle shaped and as saccular when the pouch has a narrow neck. ${ }^{6}$ In some reports fusiform coronary aneurysms have been labelled as saccular when they assume a globular configuration even though a narrow neck is not present. ${ }^{4}$ Fusiform dilatation or "coronary ectasia" is a relatively common finding, which is usually present in conjunction with atherosclerotic narrowings; the pathogenesis of such aneurysmal dilatation is thought to involve intimal disruption or to be secondary to medial disease. ${ }^{45}$ In contrast, true saccular coronary artery aneurysms are uncommon and little is known of their pathogenesis or course. The appearance of the aneurysm in this case differs from that in most large reviews of coronary aneurysms because they included cases of coronary ectasia. ${ }^{14}$ The angiographic appearance of coronary aneurysms at the site of angioplasty seems to be similar to what we found in our case. ${ }^{3}$ The 
availability of serial angiograms during the development of the aneurysm has allowed us to link the anatomical changes with the associated clinical events.

The close association between unstable angina, myocardial infarction, intimal tears, and thrombus formation has become increasingly recognised. However, our case has several unusual features. The initial angiographic appearance was of a stenosis of $<$ $50 \%$ of the luminal diameter of the proximal left anterior descending artery -an unexpected finding in a patient with unstable angina-and anterior ST changes during rest pain. Reduced density of contrast at the site of the lesion at that time suggested the presence of thrombus. The initial episodes of pain may have been caused by further local thrombus formation giving rise to a transient increase in obstruction to flow or caused by embolisation of thrombotic or atheromatous material. Embolisation seems more likely to be the mechanism of myocardial ischaemia in this case because the electrocardiographic changes persisted for days despite wide patency of the left anterior descending artery.

Embolisation of material may produce ischaemia or have more serious consequences. Platelet and fibrin microthrombi were quite common in ischaemic heart disease and in particular may be important in sudden cardiac death in young people. ${ }^{7}$

Heparin infusion would have been expected to reduce the amount of thrombus present. It is conceivable that in so doing the underlying ulcerated plaque was exposed, thus revealing the small protrusion of contrast seen on the second angiogram. The fact that the aneurysm became larger in association with further unstable angina again suggests the possibility of embolisation, perhaps of the contents of the plaque or of thrombotic material formed within it. Such mechanisms have been postulated to explain the development of coronary artery aneurysms ${ }^{5}$ but no serial angiographic studies have been reported.
The likelihood of embolisation of atheromatous material as an integral part of the sequence has also been suggested as the explanation for the finding of a high incidence of reduced left ventricular function in patients with coronary aneurysms in the absence of coronary occlusion. In our patient left ventricular function was well maintained.

The role of anticoagulants, antiplatelet treatment, or operation is uncertain, but operation has been of suggested as a means of reducing emboli and removing the risk of rupture. In the absence of current symptoms and the very low incidence of $\vec{\omega}$ atherosclerotic coronary artery rupture we elected to continue medical treatment.

\section{References}

1 Robinson FC. Aneurysms of the coronary arteries. $A m$ Heart $J$ 1985;109:129-35.

2 Anabatwi IN, de Leon JA. Arteriosclerotic aneurysms of the coronary arteries. $J$ Thorac Cardiovasc Surg 1974;68:226-8.

3 Hill JA, Margolis JR, Feldman RL, Conti CR, Pepine CJ. Coronary arterial aneurysm formation after balloon angioplasty. Am J Cardiol 1983;52:261-4.

4 Befeler B, Aranda JM, Embi A, Mullin FL, El-Sherif N, Lazzara R. Coronary artery aneurysms; study of their etiology, clinical course and effect on left ventricular function and prognosis. Am J Med 1977; 62:597-607.

5 Berkoff HA, Rowe GG. Atherosclerotic ulcerative disease and associated aneurysms of the coronary arteries. Am Heart J 1975;90:153-7.

6 Lindsay $\mathrm{J} \mathrm{Jr}$. Diseases of the aorta and vena cavae. In: Hurst JW, ed. The heart. 4th ed. New York: McGraw-Hill, 1978:1849-56.

7 El-Maraghi N, Genton E. The relevance of platelet and fibrin thromboembolism of the coronary microcirculation, with special reference to sudden cardiac death. Circulation 1980;62:936-44. 